\title{
The role of miR-766-5p in cell migration and invasion in colorectal cancer
}

\author{
BIN JIA $^{1}$, LEI XIA ${ }^{2}$ and FANG CAO ${ }^{2}$ \\ Departments of ${ }^{1}$ Gastroenterology and ${ }^{2}$ Oncology, Affiliated Hospital of Shandong University \\ of Traditional Chinese Medicine, Jinan, Shandong 250014, P.R. China
}

Received May 11, 2017; Accepted September 19, 2017

DOI: $10.3892 /$ etm.2018.5716

\begin{abstract}
Colorectal cancer (CRC) develops from the colon or rectum and is the fourth highest inducer of cancer mortality. In the present study, cancer tissues and normal tissues were extracted from patients with CRC who were treated in the Affiliated Hospital of Shandong University of Traditional Chinese Medicine (Jinan, China). Reverse transcription-quantitative polymerase chain reaction demonstrated that the expression level of miR-766-5p was significantly higher $(\mathrm{P}<0.01)$ in cancer tissue than that in normal tissue. SW480 cells were used for in vitro study and randomly separated into the miR-negative control (NC) inhibitor treatment group and miR-766-5p inhibitor treatment group. SW480 cell behaviors were evaluated. Results demonstrated that in the miR-766-5p inhibitor group, there was a decreased level of cell proliferation/migration/invasion and higher cell apoptosis compared with that in the miR-NC inhibitor group. miR-766-5p was predicted and verified to target the 3' untranslated region of suppressor of cancer cell invasion (SCAI) in SW480 cells. Protein expression levels of matrix metalloproteinase-2/phosphoinositide 3-kinase/AKT were decreased and SCAI was increased following miR-766-5p inhibitor treatment. In conclusion, the present study indicated that miR-766-5p inhibitor repressed the process of CRC by targeting SCAI.
\end{abstract}

\section{Introduction}

Colorectal cancer (CRC), known as either bowel cancer or colon cancer, develops from the colon or rectum (1). CRC predominantly results from lifestyle and old age, with very few cases of CRC resulting from genetic disorders (2). CRC accounts for $13 \%$ of all cancer cases worldwide (3). In 2012, 1.4 million

Correspondence to: Dr Fang Cao, Department of Oncology, Affiliated Hospital of Shandong University of Traditional Chinese Medicine, 16369 Jingshi Road, Jinan, Shandong 250014, P.R. China E-mail: caofang7894@sina.com

Key words: colorectal cancer, suppressor of cancer cell invasion, miR-766-5p new cases of CRC were diagnosed and 694,000 mortalities as a result of CRC were recorded (4). CRC is the fourth greatest cause of cancer-related mortality after lung, stomach and liver cancer (5).

The purpose of CRC treatment is cure or palliation, and this depends mainly on factors including the patient's health status, preference and tumor stage (6). Currently, a combination of surgery, radiation therapy, chemotherapy and targeted therapy is commonly adopted for the treatment of CRC (1). A cure may be achieved by surgery if CRC is detected at an earlier stage, while palliation may be directed through relieving CRC-related symptoms when CRC is diagnosed later (7). It is necessary to explore effective treatments for patients with CRC.

There are types of microRNA (miRNA), which are $\sim 22$ nucleotides long, that inhibit protein expression via targeting its coding gene (8). Generally, miRNA in animals are complementary to a site in the $3^{\prime}$ untranslated region (UTR) of their target gene (9). On account of the involvement of miRNA in eukaryotic cell function, the dysregulation of miRNA is correlated with disease (10) and cancer (11). Notably, miRNA-based therapies have been reported to be a potential for cancer treatment (12). miR-766-5p has been demonstrated to be overexpressed in CRC and promote the cell proliferation of SW480 cells (13). However, the role of miR-766-5p in cell migration and invasion of CRC has not been reported. The present study aimed to investigate the aforementioned question.

\section{Materials and methods}

Clinical samples. A total of 31 pairs of tumor tissues and adjacent tissues, which were at least 2-cm distal to tumor margins, were collected from 31 patients with CRC who were admitted to the Affiliated Hospital of Shandong University and underwent surgery between January 2014 and January 2015. There were 15 male patients and 16 female patients with the age ranged from 47 to 73 years old. Following collection, tissues were quickly frozen and stored in $-196^{\circ} \mathrm{C}$ liquid nitrogen. None of the patients received neoadjuvant therapy. The present study was approved by the Ethics Committee of the Affiliated Hospital of Shandong University of Traditional Chinese Medicine (Jinan, China). Informed consent was obtained from each patient. Tissues were used for the detection of mRNA expression levels. 
Cell culture. The human CRC cell line, SW480, was purchased from Nanjing KGI Biotechnology (Nanjing, China) and cultured in Dulbecco's modified Eagle's medium supplemented with $10 \%$ fetal bovine serum (Invitrogen; Thermo Fisher Scientific, Inc., Waltham, MA, USA), $100 \mathrm{U} / \mathrm{ml}$ penicillin and $100 \mu \mathrm{g} / \mathrm{ml}$ streptomycin, and incubated in a humidified chamber at $37^{\circ} \mathrm{C}$ with an atmosphere of $5 \% \mathrm{CO}_{2}$ and $95 \%$ air.

Plasmid transfection. SW480 cells were seeded into 24-well plates at a density of $1 \times 10^{5}$ cells/well. Cells were randomly divided into two groups: miR-766-5p inhibitor group and miR-negative control (NC) inhibitor group. Transfection of SW480 cells with $30 \mu \mathrm{M}$ miR-766-5p inhibitor or miR-NC inhibitor (GeneCopoeia, Rockville, MD, USA) was conducted by Lipofectamine 2000 transfection reagent (Thermo Fisher Scientific, Inc.) in accordance with the manufacturer's protocol. SW480 cells were collected $48 \mathrm{~h}$ after transfection for subsequent experiments.

Luciferase activity assay. Suppressor of cancer cell invasion (SCAI) was predicted to be recognized by miR-766-5p using TargetScan (http://www.targetscan.org/), and the recombinant plasmids of pmir-SCAIwt-3'UTR and pmir-SCAImut-3'UTR (Promega Corporation, Madison, WI, USA) were constructed. SW480 cells were incubated for $24 \mathrm{~h}$ at $37^{\circ} \mathrm{C}$, followed by co-transfection of miR-766-5p inhibitor and pmir-SCAIwt-3'UTR or pmir-SCAImut-3'UTR using Lipofectamine 2000 reagents. After 24 h, luciferase activities were assessed using the Dual-Luciferase Reporter Assay System (Promega Corporation). Renilla luciferase was used as an internal control.

Cell viability analysis. SW480 cells $\left(2 \times 10^{3}\right.$ cells/well) were seeded onto 96 -well plates. Cell viability was evaluated with a cell counting kit-8 (CCK-8; Dojindo Molecular Technologies, Inc., Rockville, MD, USA), according to the manufacturer's protocol, at 24,48 or $72 \mathrm{~h}$ after transfection with miR-766-5p inhibitor or miR-NC inhibitor. Absorbance was read at $490 \mathrm{~nm}$ with an iMARK plate reader (Bio-Rad Laboratories, Inc., Hercules, CA, USA).

Cell apoptosis analysis. Transfected SW480 cells were seeded into 12 -well plates at the density of $2 \times 10^{5} /$ well, cultured for $48 \mathrm{~h}$ at $37^{\circ} \mathrm{C}$ and collected via centrifugation at $23,200 \mathrm{x} \mathrm{g}$ for $5 \mathrm{~min}$ at $4^{\circ} \mathrm{C}$. Following three washes with PBS, SW480 cells were re-suspended in $100 \mu \mathrm{l}$ binding buffer, stained with annexin V-fluorescein isothiocyanate and propidium iodide (Roche Diagnostics, Basel, Switzerland) for $15 \mathrm{~min}$ at room temperature in the dark. The rate of cell apoptosis was determined by flow cytometry (Beckman Coulter, Inc., Miami, FL, USA) in $1 \mathrm{~h}$. The cell number at each phase was analyzed suing FloJo software (Version 7.6.3, Treestar, Inc., Ashland, OR, USA).

Cell invasion assay. Cell invasiveness of SW480 cells was evaluated by Transwell chambers, which were coated with Matrigel (BD Biosciences, Franklin Lakes, NJ, USA). Transfected SW480 cells were placed in Transwell upper chambers that were coated with gelatin. Functioning as a chemoattractant, $0.2 \%$ BSA containing fibronectin (Bio-Techne, Shanghai,
China) $(10 \mu \mathrm{g} / \mathrm{ml})$ was added to the bottom chamber. For the invasion assay, $100 \mu \mathrm{l}$ transfected SW480 cells at a concentration of $1 \times 10^{5}$ cells were cultured in the upper chamber in serum-free Dulbecco's modified Eagle's medium at $37^{\circ} \mathrm{C}$ in $5 \% \mathrm{CO}_{2}$ humidified air. A total of $16 \mathrm{~h}$ later, non-invading cells on the upper chambers were removed with a cotton swab, and invading cells that reached the bottom chambers were fixed with $70 \%$ ethanol for $15 \mathrm{~min}$ at room temperature and stained by Hemacolor ${ }^{\circledR}$ Rapid staining solution (Merck KGaA, Darmstadt, Germany) at room temperature for $30 \mathrm{~min}$. The number of invasive cells was counted under a light microscope (magnification, $\mathrm{x} 400$ ).

Cell migration assay. Cell migration was evaluated using a wound healing assay. Transfected SW480 cells were cultured in an incubator at $37^{\circ} \mathrm{C}$ with $5 \% \mathrm{CO}_{2}$. After incubation for $24 \mathrm{~h}$, a wound gap was created using a 20- $\mu$ l pipette tip. Transfected SW480 cells were further cultured for $24 \mathrm{~h}$. Thereafter, wounded monolayers were photographed with an inverted microscope (magnification, $\mathrm{x} 400$ ).

Western blotting. Protein lysates were obtained with radioimmunoprecipitation assay buffer (Beyotime Institute of Biotechnology, Shanghai, China). Concentration of protein samples were determined using the BCA method (Beyotime Institute of Biotechnology). Protein samples (15 $\mu \mathrm{g} /$ lane) were first separated by $10 \%$ SDS-PAGE and transferred, at $4^{\circ} \mathrm{C}$, onto polyvinylidene difluoride membranes followed by blocking by $5 \%$ skim milk at room temperature for $1 \mathrm{~h}$. Membranes were first incubated overnight with primary antibodies (SCAI, 12892, 1:1,000, Cell Signaling Technology, Inc., Danvers, MA, USA; MMP2, ab37150, 1:1,000, Abcam, Cambridge, UK; p-PI3K, ab151549, 1:1,000, Abcam; p-AKT, 4060, 1:1,000, Cell Signaling Technology, Inc.; GAPDH, $5174,1: 1,000$, Cell Signaling Technology, Inc.) at $4^{\circ} \mathrm{C}$, then treated with horseradish peroxidase-conjugated secondary antibodies (Santa Cruz Biotechnology, Inc., Dallas, TX, USA) for $2 \mathrm{~h}$ at room temperature. Blots were visualized with an enhanced chemiluminescent kit (Beyotime Institute of Biotechnology).

Reverse transcription-quantitative polymerase chain reaction $(R T-q P C R)$. Total RNA was extracted from SW480 cells at $48 \mathrm{~h}$ after transfection and from tissue samples from patients with CRC using TRIzol (Thermo Fisher Scientific, Inc.), and RT-qPCR for miRNA was executed using a one-step TaqMan miRNA reverse-transcription kit (Thermo Fisher Scientific, Inc.). The following thermocycling conditions were used: $95^{\circ} \mathrm{C}$ for $40 \mathrm{sec}$ at initial denaturation, followed by 45 cycles for $30 \mathrm{sec}$ at $95^{\circ} \mathrm{C}$ for denaturation and $30 \mathrm{sec}$ at $58^{\circ} \mathrm{C}$, with the final extension at $72^{\circ} \mathrm{C}$ for $35 \mathrm{sec}$. The relative mRNA expression levels of miR-766 (normalized to U6) and SCAI (normalized to GAPDH), were calculated using the $2^{-\Delta \Delta \mathrm{Cq}}$ method (14). The primer sequences were as followed: GAPDH, forward 5'-AGCCTCCCGCTTCGCTCTCTGC-3' and reverse 5'-ACCAGGCGCCCAATACGACCAAA-3'; U6, forward 5'-GCTTCGGCAGCACATATACTAAAAT-3' and reverse 5'-CGCTTCACGAATTTGCGT-3'; SCAI, forward 5'-CGG GAAACACGAAATTATCC-3' and reverse 5'-GCTTCTGGA GATGAGGATTCTC-3'; and miR-766, forward 5'-TCGAGT 
Table I. Patient characteristics.

\begin{tabular}{lcc}
\hline Factor & $\mathrm{n}$ & P-value \\
\hline Gender & 15 & \\
$\quad$ Male & 16 & $>0.05$ \\
Female & & \\
Age, years & 17 & \\
$<60$ & 14 & $>0.05$ \\
$\geq 60$ & & \\
Tumor size, cm & 11 & $<0.05$ \\
$<5$ & 20 & \\
$\geq 5$ & & $<0.05$ \\
Tumor differentiation degree & 9 & \\
Low degree & 22 & $<0.05$ \\
Intermediate degree & & \\
Metastasis & 10 & \\
No & 21 & \\
Yes &
\end{tabular}

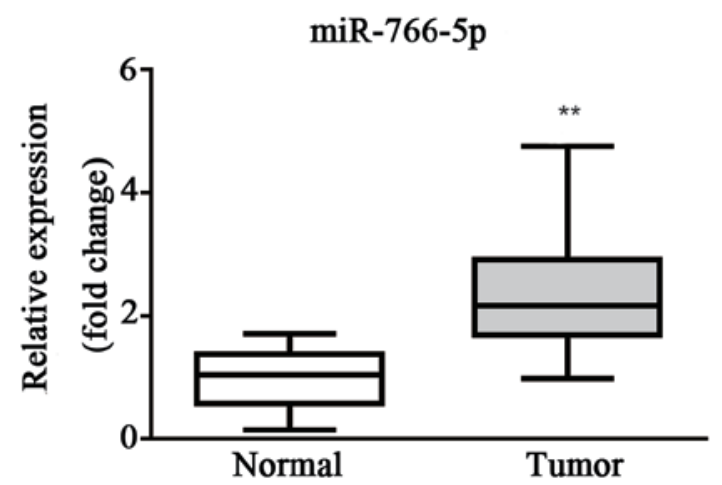

Figure 1. miR-766-5p expression is elevated in colorectal cancer tissues. Reverse transcription-quantitative polymerase chain reaction results demonstrated that the expression level of miR-766-5p was significantly higher in cancer tissue than in healthy (normal) tissue. ${ }^{* *} \mathrm{P}<0.01$ vs. normal tissues.

\section{ACTTGAGATGGAGTTTT-3' and reverse 5'-GGCCGCGTT GCAGTGAGCCGAG-3'.}

Statistical analysis. All data were presented as the mean \pm standard deviation. The analysis was performed using GraphPad Prism 5.0 (GraphPad Software, Inc., La Jolla, CA, USA). Differences between two groups were analyzed using a Student's t-test, and differences between three or more groups were analyzed using one-way analysis of variance followed by Newman-Keuls analysis. $\mathrm{P}<0.05$ was considered to indicate a statistically significant difference.

\section{Results}

miR-766-5p expression is higher in CRC tissues than normal tissues. The miR-766-5p expression level in patient tissues was first evaluated by RT-qPCR. Results demonstrated that the expression level of miR-766-5p was significantly higher in cancer tissue than the level in healthy tissue, which suggests
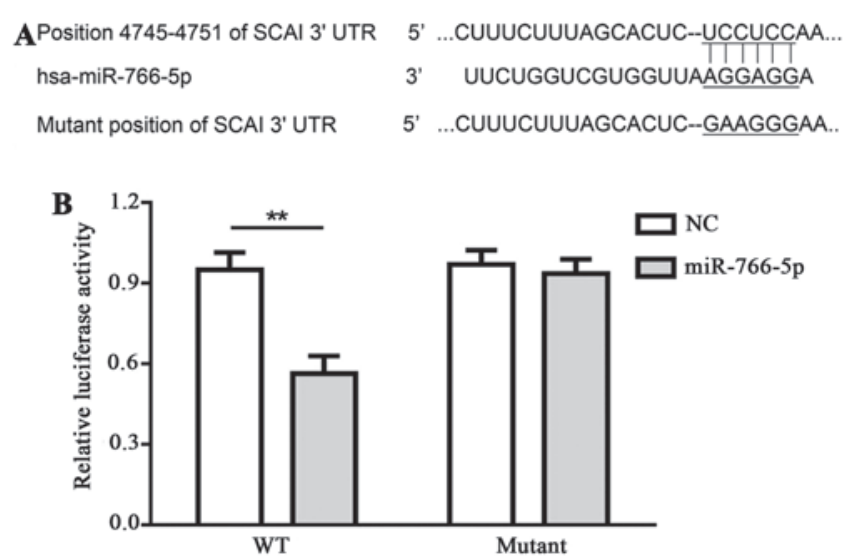

Figure 2. miR-766-5p targets the 3'UTR of SCAI in SW480 cells (A) Binding position of SCAI 3'UTR and miR-766-5p, and the mutant position of SCAI 3'UTR. (B) miR-766-5p inhibitor significantly decreased the luciferase activity in SW480 cells transfected with pmir-SCAIwt-3'UTR but not pmir-SCAImut-3'UTR. ${ }^{* *} \mathrm{P}<0.01$ as indicated as cells transfected with pmir-SCAIwt-3'UTR and miR-766-5p inhibitor vs. cells transfected with pmir-SCAIwt-3'UTR and miR-NC inhibitor. UTR, untranslated region; SCAI, suppressor of cancer cell invasion; WT, wild type-SW480 cells were transfected with pmir-SCAIwt-3'UTR; mutant, SW480 cells were transfected with pmir-SCAImut-3'UTR; NC, negative control.

that miR-766-5p may be a promoter during the progression of CRC (Fig. 1).

Interaction between miR-766-5p and its target, SCAI, in SW480 cells. TargetScan was applied for the prediction of interaction between miR-766-5p and its potential targets. SCAI demonstrated a relative higher score when compared with other potential targets, thus, SCAI was selected in the present study. Binding sequences between miR-766-5p and SCAI 3'UTR, as well as the mutant sequences, are presented in Fig. 2A. Luciferase activity in SW480 cells that were transfected with pmir-SCAIwt-3'UTR and miR-766-5p inhibitor was decreased compared with cells transfected with pmir-SCAIwt-3'UTR and miR-NC inhibitor, while there was no significant difference in cells transfected with pmir-SCAImut-3'UTR and miR-766-5p inhibitor in comparison with cells transfected with pmir-SCAImut-3'UTR and miR-NC inhibitor (Fig. 2B). These results demonstrate that miR-766-5p targets and binds with the 3'UTR of SCAI.

Characteristics of patients with CRC. Patients' age, sex, tumor size, tumor differentiation degree and metastasis are presented in Table I. There were no significant differences in patient age or gender; however, there were significant differences in tumor size, differentiation degree and metastasis.

SCAI expression is lower in CRC tissues than in normal tissues. SCAI expression level in patient tissues was determined by RT-qPCR. Results demonstrated that the SCAI expression level was significantly lower in cancer tissue than in healthy tissue (Fig. 3).

Effects of miR-766-5p inhibitor on miR-766-5p/SCAI expression in SW480 cells. For in vitro experiments, SW480 cells were transfected with miR-NC inhibitor and miR-766-5p inhibitor. The cells in the two different groups were used for 


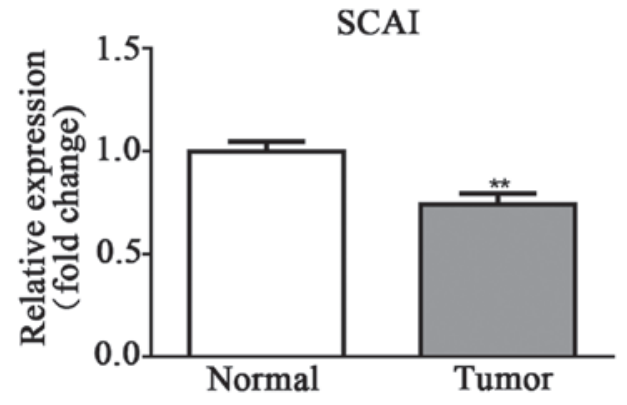

Figure 3. SCAI expression is decreased in colorectal cancer tissues. Results of reverse transcription-quantitative polymerase chain reaction demonstrated that the SCAI expression level was significantly lower in tumor tissue than in normal tissue. ${ }^{* *} \mathrm{P}<0.01$ vs. normal tissues. SCAI, suppressor of cancer cell invasion.
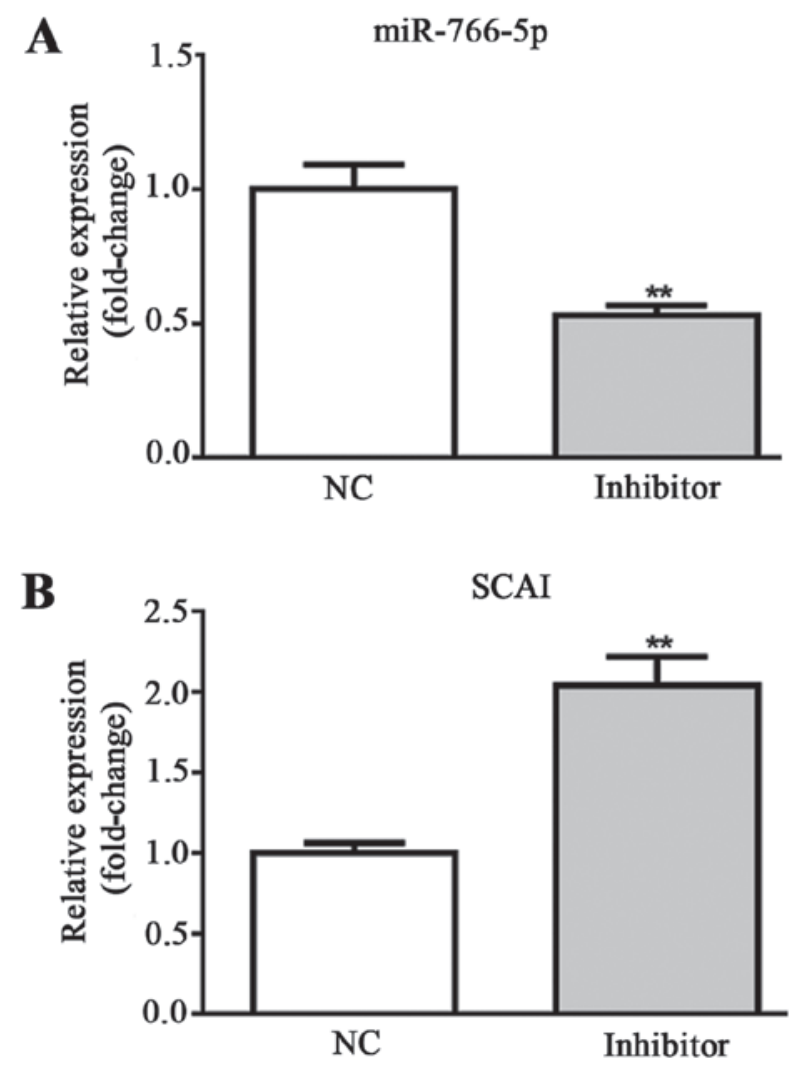

Figure 4. miR-766-5p inhibitor reduces miR-766-5p and elevates SCAI expression in SW480 cells. Results of reverse transcription-quantitative polymerase chain reaction demonstrated that, compared with the NC group, (A) miR-766-5p expression was significantly decreased in the miR-766-5p inhibitor group. Additionally, when compared with the NC group, (B) SCAI expression was significantly upregulated in the miR-766-5p inhibitor group ${ }^{* *} \mathrm{P}<0.01$ vs. NC group. NC, negative control; SCAI, suppressor of cancer cell invasion.

the detection of miR-766-5p and SCAI expression levels by RT-qPCR. Results demonstrated that, compared with the NC group, miR-766-5p expression was significantly decreased and SCAI expression was significantly increased in the miR-766-5p inhibitor group (Fig. 4A and B). These results suggest that SW480 cells were successfully transfected with miR-766-5p inhibitor. The effects of miR-766-5p on cell behaviors and the corresponding molecules were subsequently determined.

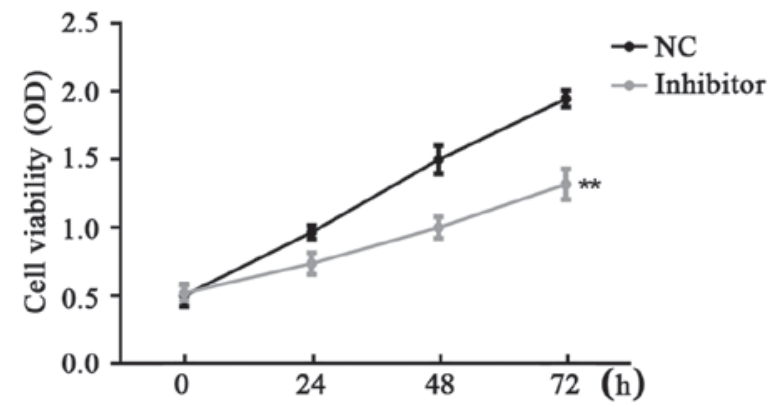

Figure 5. miR-766-5p inhibitor reduces SW480 cell viability. At 24, 48 and $72 \mathrm{~h}$ after transfection, miR-766-5p inhibitor decreased SW480 cell proliferation in comparison with the NC group. ${ }^{* *} \mathrm{P}<0.01$ vs. NC group at 24,48 and $72 \mathrm{~h}$. NC, negative control; OD, optical density.

Examination of SW480 cell viability. CCK-8 assay was used for the examination of SW480 cell viability in the different groups. Results demonstrated that miR-766-5p inhibitor significantly inhibited SW480 cell proliferation compared with the $\mathrm{NC}$ group at 24,48 and $72 \mathrm{~h}$ after transfection (Fig. 5).

Detection of SW480 cell apoptosis. Flow cytometry was used for the examination of SW480 cell apoptosis in the different groups. Results demonstrated that miR-766-5p inhibitor significantly promoted SW480 cell apoptosis (35.98\%) compared with the NC group (17.79\%) (Fig. 6A and B).

Evaluation of SW480 cellmigration/invasion. A wound healing assay was used for the examination of SW480 cell migration, while Transwell assays were used for the examination of SW480 cell invasion in different groups. Results demonstrated that the miR-766-5p inhibitor markedly inhibited SW480 cell migration/invasion compared with that observed in the $\mathrm{NC}$ group (Fig. 7A and B).

Determination of protein levels of SCAI, phosphoinositide 3-kinase (PI3K)/AKT and matrix metalloproteinase-2 (MMP2). In comparison with the NC group, protein expression levels of MMP2 and phosphorylated (p)-PI3K/AKT were decreased, while SCAI was increased following treatment with miR-766-5p inhibitor. GAPDH was used as an internal control (Fig. 8). Taken together, these results indicated that, miR-766-5p inhibitor repressed the activation of $\mathrm{p}-\mathrm{PI} 3 \mathrm{~K} / \mathrm{p}-\mathrm{AKT}$ signaling by targeting SCAI.

\section{Discussion}

miRNA function as oncogenes or tumor suppressors $(15,16)$. Mounting evidence suggests that miRNA serve fundamental roles in cell proliferation, migration and invasion (17-19). Aberrant expression of miRNA has been increasingly explored (17-19). Furthermore, miR-766-5p was reported to be overexpressed in CRC and promote the cell proliferation of SW480 cells (13). The present study aimed to investigate the role of miR-766-5p in SW480 cells.

SCAI 3'UTR was verified to be recognized by miR-766-5p. The present study demonstrated that SCAI was deregulated in tumor tissues compared with normal tissues, which was 
$\mathbf{A}$

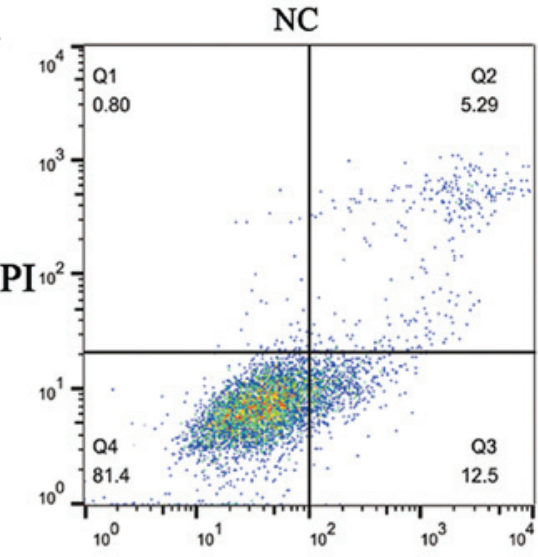

Annexin V-FITC

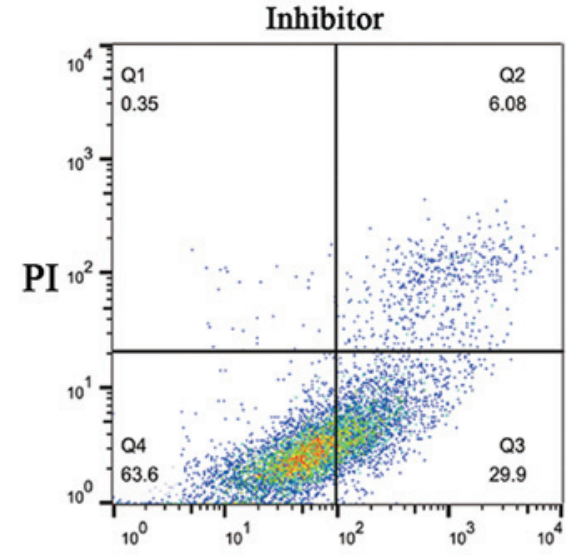

Annexin V-FITC

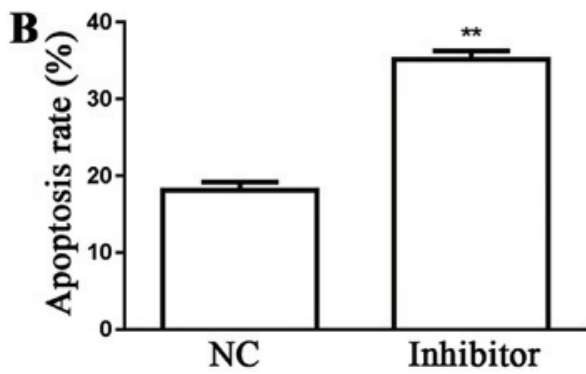

Figure 6. miR-766-5p inhibitor induces SW480 cell apoptosis. (A) Compared with the NC group, the SW480 cell apoptosis rate was higher in the miR-766-5p inhibitor group. (B) The statistical data are also presented. ${ }^{* *} \mathrm{P}<0.01$ vs. NC group. NC, negative control; PI, propidium iodide; FITC, fluorescein isothiocyanate.

$\mathbf{A}$

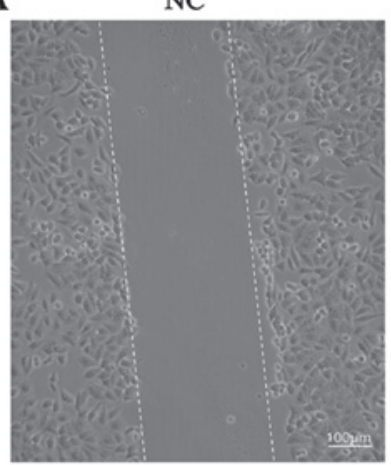

B

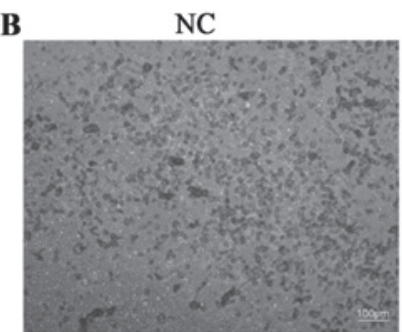

Inhibitor

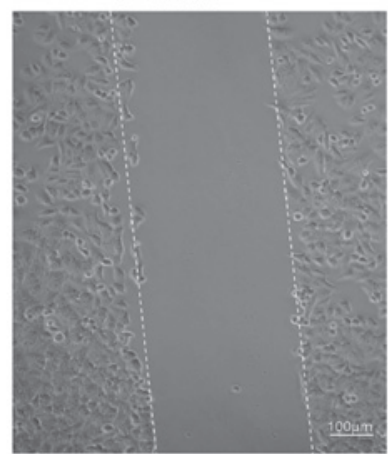

Inhibitor

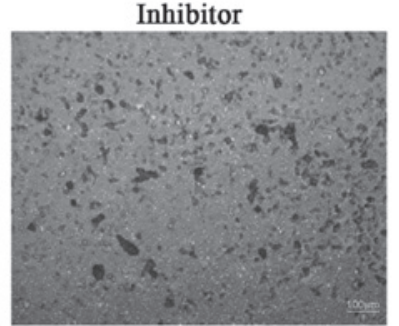

Figure 7. miR-766-5p inhibitor induces SW480 cell migration/invasion. miR-766-5p inhibitor notably inhibited SW480 cell (A) migration (B) invasion compared with that observed in the NC group. Scale bar, $100 \mu \mathrm{m}$. Stained was conducted using Hemacolor ${ }^{\circledR}$ Rapid staining solution. NC, negative control.

consistent with a previous study that also reported the decrease of SCAI in human cancer, implying a tumor suppressive role $(20,21)$. In the present study, western blotting revealed that,

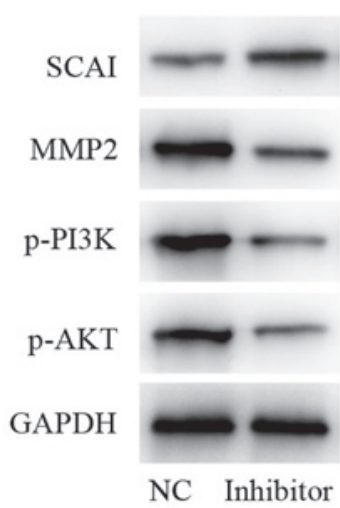

Figure 8. miR-766-5p inhibitor decreases the PI3K/AKT and MMP2 expression level, and increases the SCAI expression level. Protein levels of MMP2 and p-PI3K/AKT were lower, while SCAI was higher in the miR-766-5p inhibitor group than in the NC group. NC, negative control; SCAI, suppressor of cancer cell invasion; MMP2, matrix metalloproteinase-2; $\mathrm{p}$, phosphorylated; PI3K, phosphoinositide 3-kinase.

following miR-766-5p inhibitor administration, SCAI protein level was upregulated in SW480 cells in comparison with the level in the NC group, demonstrating the interaction between miR-766-5p and SCAI. RNA-mediated interference-induced knockdown of SCAI has been demonstrated to increase cell migration ability (22). However, the effects of SCAI on SW480 cell behaviors were yet to be investigated.

Metastasis, the major cause of cancer mortality, is a complex process caused by changes in proto-oncogenes and tumor suppressor genes, which are implicated in cancer cell 
growth, migration, invasion and angiogenesis $(23,24)$. As acknowledged, cell migration and invasion serve pivotal roles in angiogenesis, tissue repair and immune response, which are all deregulated in cancer cells $(25,26)$. In the present study, the influence of miR-766-5p inhibitor on SW480 cell proliferation, apoptosis, migration and invasion was investigated. Results demonstrated that, miR-766-5p inhibitor treatment reduced cell proliferation, migration and invasion ability, and induced cell apoptosis of SW480 cells. Taken together, the miR-766-5p inhibitor slowed down the process of CRC. The present study also explored whether there are other molecules that are influenced by miR-766-5p.

MMP family proteolytic enzymes are indispensable for the remodeling of extracellular matrix, of which the degradation is a prerequisite for the invasion and metastasis of cancer $(27,28)$. In the present study, it was demonstrated that the protein expression level of MMP2 was notably decreased following miR-766-5p inhibitor treatment compared to that following treatment with the NC. Additionally, the PI3K/AKT/mammalian target of rapamycin pathway was reported to be a potential target for prevention of CRC $(29,30)$. In the present study, protein expression levels of p-PI3K and p-AKT were also found to be markedly decreased following miR-766-5p inhibitor treatment compared to the levels in the NC group.

In conclusion, treatment with a miR-766-5pinhibitor reduces cell proliferation, migration, invasion, and MMP2/PI3K/AKT protein expression in the SW480 CRC cell line, and induces cell apoptosis via targeting SCAI. The findings of the present study propose a possible treatment target for CRC.

\section{References}

1. National Cancer Institute (NCI): Colon Cancer Treatment (PDQ ${ }^{\circledR}$ )-Patient Version. NCI, Bethesda, MD, 2017. https:// www.cancer.gov/types/colorectal/patient/colon-treatment-pdq. Updated February 27, 2017.

2. IARC: World Cancer Report 2014: World Health Organization. Chapter 5: 5, 2014.

3. Parkin DM, Bray F, Ferlay J and Pisani P: Global cancer statistics, 2002. CA Cancer J Clin 55: 74-108, 2005.

4. IARC: World Cancer Report 2014: World Health Organization. Chapter 1: 1, 2014.

5. Brody H. Colorectal cancer. Nature 521: S1, 2015

6. Stein A, Atanackovic D and Bokemeyer C: Current standards and new trends in the primary treatment of colorectal cancer. Eur J Cancer 47 (Suppl 3): S312-S314, 2011.

7. Cunningham D, Atkin W, Lenz HJ, Lynch HT, Minsky B, Nordlinger B and Starling N: Colorectal cancer. Lancet 375: 1030-1047, 2010

8. Bartel DP: MicroRNAs: Genomics, biogenesis, mechanism, and function. Cell 116: 281-297, 2004.

9. Wang XJ, Reyes JL, Chua NH and Gaasterland T: Prediction and identification of Arabidopsis thaliana microRNAs and their mRNA targets. Genome Biol 5: R65, 2004.
10. Mraz M and Pospisilova S: MicroRNAs in chronic lymphocytic leukemia: From causality to associations and back. Expert Rev Hematol 5: 579-581, 2012.

11. Musilova K and Mraz M: MicroRNAs in B cell lymphomas: How a complex biology gets more complex. Leukemia 29: 1004-1017, 2015.

12. Trang P, Weidhaas JB and Slack FJ: MicroRNAs as potential cancer therapeutics. Oncogene 27 (Suppl 2): S52-S57, 2008.

13. Li YC, Li CF, Chen LB, Li DD, Yang L, Jin JP and Zhang B: MicroRNA-766 targeting regulation of SOX6 expression promoted cell proliferation of human colorectal cancer. Onco Targets Ther 8: 2981-2988, 2015.

14. Livak KJ and Schmittgen TD: Analysis of relative gene expression data using real-time quantitative PCR and the 2(-Delta Delta C(T)) method. Methods 25: 402-408, 2001.

15. Esquela-Kerscher A and Slack FJ: Oncomirs-microRNAs with a role in cancer. Nat Rev Cancer 6: 259-269, 2006.

16. Osada $\mathrm{H}$ and Takahashi T: MicroRNAs in biological processes and carcinogenesis. Carcinogenesis 28: 2-12, 2007.

17. Shen F, Cai WS, Feng Z, Li JL, Chen JW, Cao J and Xu B: miR-492 contributes to cell proliferation and cell cycle of human breast cancer cells by suppressing SOX7 expression. Tumour Biol 36: 1913-1921, 2015.

18. Zhang Z, Sun J, Bai Z, Li H, He S, Chen R and Che X: MicroRNA-153 acts as a prognostic marker in gastric cancer and its role in cell migration and invasion. Onco Targets Ther 8: 357-364, 2015.

19. Li X, Wang J, Jia Z, Cui Q, Zhang C, Wang W, Chen P, Ma K and Zhou C: miR-499 regulates cell proliferation and apoptosis during late-stage cardiac differentiation via Sox6 and cyclin D1. PLoS One 8: e74504, 2013.

20. Brandt DT, Baarlink C, Kitzing TM, Kremmer E, Ivaska J, Nollau P and Grosse R: SCAI acts as a suppressor of cancer cell invasion through the transcriptional control of beta1-integrin. Nat Cell Biol 11: 557-568, 2009.

21. Chen X, Hu W, Xie B, Gao H, Xu C and Chen J: Downregulation of SCAI enhances glioma cell invasion and stem cell like phenotype by acti-vating Wnt $/ \beta$-catenin signaling. Biochem Biophys Res Commun 448: 206-211, 2014.

22. Juliano R: SCAI blocks MAL-evolent effects on cancer cell invasion. Nat Cell Biol 11: 540-542, 2009.

23. Turajlic $\mathrm{S}$ and Swanton C: Metastasis as an evolutionary process. Science 352: 169-175, 2016.

24. Brábek J, Mierke CT, Rösel D, Veselý P and Fabry B: The role of the tissue microenvironment in the regulation of cancer cell motility and invasion. Cell Commun Signa 8: 22, 2010.

25. Haeger A, Wolf K, Zegers MM and Friedl P: Collective cell migration: Guidance principles and hierarchies. Trends Cell Biol 25: 556-566, 2015.

26. Clark AG and Vignjevic DM: Modes of cancer cell invasion and the role of the microenvironment. Curr Opin Cell Biol 36: 13-22, 2015.

27. Galliera E, Tacchini L and Corsi Romanelli MM: Matrix metalloproteinases as biomarkers of disease: Updates and new insights. Clin Chem Lab Med 53: 349-355, 2015.

28. Cathcart J, Pulkoski-Gross A and Cao J: Targeting matrix metalloproteinases in cancer: Bringing new life to old ideas. Genes Dis 2: 26-34, 2015.

29. Ballard-Barbash R, Friedenreich CM, Courneya KS, Siddiqi SM, McTiernan A and Alfano CM: Physical activity, biomarkers, and disease outcomes in cancer survivors: A systematic review. J Natl Cancer Inst 104: 815-840, 2012.

30. Pandurangan AK: Potential targets for prevention of colorectal cancer: A focus on PI3K/Akt/mTOR and Wnt pathways. Asian Pac J Cancer Prev 14: 2201-2205, 2013. 